\title{
Volumetric modulated arc therapy (VMAT) for hippocampal-avoidance whole brain radiation therapy: planning comparison with Dual-arc and Split-arc partial-field techniques
}

\author{
Adams Hei Long Yuen ${ }^{1,2^{*}}$, Po Man Wu', Alex Kai Leung Li ${ }^{2}$ and Philip Chung Yin Mak
}

\begin{abstract}
Background: Although whole brain radiation therapy (WBRT) provides palliation and prophylaxis, reduces local recurrence probability and improves overall survival, it is evident that WBRT is associated with neurocognitive deficits due to radiation induced damage of the hippocampus. Therefore, minimizing hippocampal dose to the least possible level is of high clinical relevance. In dual-arc conventional volumetric modulated arc therapy (dacVMAT), the large irradiation field for whole brain planned target volume (PTV) requires a wide jaw opening in which substantial low dose volume to the hippocampus may be produced due to suboptimal multi-leaf collimator (MLC) movements. The present study investigates the potential of a radiation therapy technique with split-arc and reduced field size, namely split-arc partial-field volumetric modulated arc therapy (sapf-VMAT) to spare the hippocampus during WBRT.
\end{abstract}

Methods: Computed tomography and magnetic resonance images of 20 patients with brain metastases were retrieved in this retrospective planning study. The hippocampus was manually delineated by single radiation oncologist strictly following the RTOG 0933 atlas definition. Plans delivering $30 \mathrm{~Gy}$ in 10 fractions were generated for each patient using dac-VMAT and sapf-VMAT. Dosimetric parameters from both techniques were compared by paired t-test.

Results: The results demonstrated that radiation dose to the hippocampus was significantly reduced using sapfVMAT relative to dac-VMAT plans. sapf-VMAT $\left(7.86 \mathrm{~Gy}, p=0.001\right.$ ) had significantly lowered average $\mathrm{D}_{100 \%}$ compared to dac-VMAT (9.23 Gy). Decrease in hippocampus $D_{\max }$ using sapf-VMAT (13.23 Gy, $p=0.001$ ) was statistically significant when compared to dac-VMAT $(16.33 \mathrm{~Gy})$. The resulting mean dose to the hippocampus was 9.16 Gy for the for sapf-VMAT. Mean dose of sapf-VMAT was significantly lower than dac-VMAT (10.85 Gy, $p<0.05)$. In both eyes, sapf-VMAT demonstrated significantly lower $D_{\text {mean }}$ compared to dac-VMAT $(p<0.05)$. Whole brain PTV coverage was not compromised in both techniques.

Conclusion: sapf-VMAT has demonstrated significant dose reduction to the hippocampus and both eyes compared to dac-VMAT.

Keywords: Hippocampal sparing, Partial-field, Split-arc, Volumetric modulated arc therapy, Whole brain radiation therapy, Neurocognitive deficit

\footnotetext{
* Correspondence: yhladams@hotmail.com

'Department of Diagnostic Radiology and Clinical Oncology, Li Ka Shing

Faculty of Medicine, The University of Hong Kong, Pokfulam, Hong Kong

Special Administrative Region, China

2Oncology Centre, St. Teresa's Hospital, 327 Prince Edward Road, Hong Kong

Special Administrative Region, China
}

(c) The Author(s). 2020 Open Access This article is distributed under the terms of the Creative Commons Attribution 4.0 International License (http://creativecommons.org/licenses/by/4.0/), which permits unrestricted use, distribution, and reproduction in any medium, provided you give appropriate credit to the original author(s) and the source, provide a link to the Creative Commons license, and indicate if changes were made. The Creative Commons Public Domain Dedication waiver (http://creativecommons.org/publicdomain/zero/1.0/) applies to the data made available in this article, unless otherwise stated. 


\section{Introduction}

Although whole brain radiation therapy (WBRT) provides palliation and prophylaxis, reduces local recurrence probability and improves overall survival [1-5], it is evident that WBRT is associated with neurocognitive deficits [6-10] due to radiation induced damage of neural stem cell (NSC) compartment in the hippocampus [11-14]. It is hypothesized that the NSCs in the hippocampus are exquisitely radiosensitive, radiation inflammation causes alteration of the microenvironment and subsequently forces premature differentiation of neuronal progenitor cells and adoption of glial fates [15]. Previous published clinical study of Gondi et al. [16] has demonstrated that dose to $100 \%$ volume $\left(D_{100 \%}\right)$ of the hippocampus exceeds $9 \mathrm{~Gy}$ and maximum dose $\left(\mathrm{D}_{\max }\right)$ of the hippocampus exceeds $16 \mathrm{~Gy}$ in WBRT treatment course of $30 \mathrm{~Gy}$ in 10 fractions were associated with impair memory function. In addition, accumulated preclinical and clinical data have also suggested that neurocognitive deficits manifests at much lower radiation doses than previously expected (less than $10 \mathrm{~Gy}$ ) [17]. Minimizing the radiation dose to the least possible level is of high clinical relevance since increased radiation dose to $D_{100 \%}$ and maximum dose of the hippocampus corresponded to greater decline in memory function [16, 18]. It leads to the hypothesis that hippocampal sparing in patients receiving WBRT might delay or reduce the onset, and/or severity of neurocognitive deficit.

Radiation Therapy Oncology Group (RTOG) 0933 is a single-arm phase II clinical trial that studies the effectiveness of hippocampal sparing in WBRT and has demonstrated promising results in terms of memory preservation using the dose criteria (Table 1) in the protocol [16]. In the meantime, dosimetric characteristics of dual-arc conventional volumetric modulated arc therapy (dac-VMAT) in WBRT with hippocampal sparing have been reported in previous studies following RTOG 0933 criteria [19-21]. The large irradiation field of dac-VMAT for whole brain planned target volume (PTV) required a wide jaw opening which may result in suboptimal multi-leaf collimator (MLC) movements as described in previous publication [22]: (1) Hardware restrictions for MLC movements; (2) Restricted MLC velocity from one gantry angle to another; (3) MLC may reach their limit of travelling distance when they are attempting to move to the distal part of the PTV. In extreme case, the MLC may not be able to shield the desire organs-at-risk (OARs) in distal part of PTV.

In order to prevent suboptimal MLC movements during hippocampal sparing, Shen et al. [23] has employed the partial-field technique in volumetric modulated arc therapy (VMAT) for WBRT and has reported reduced hippocampal dose; however, exact doses to the other OARs have not been described. In fact, radiationinduced toxicity to the other OARs, including the eyes, during WBRT have been described in previous publications with negative impact on patients' quality of life [24, 25]. Therefore, radiation dose to the other adjacent OARs should not be overlooked and should also be considered during treatment planning of WBRT with hippocampal sparing.

Until recently, several researchers have employed both split-arc and partial-field technique together to eliminate scatter radiation and MLC limitations in VMAT planning. This technique is beneficial in sparing adjacent OARs in breast cancer [26], cervical [27, 28], anal [28, 29], and vaginal cancer [28]. To the best of the author's knowledge, the formal literature is devoid of any reference to the application of both split-arc and partial-field technique in VMAT (sapf-VMAT) for WBRT with hippocampal sparing. In the present study, the dosimetric effect of the sapf-VMAT is studied to verify its sparing ability to hippocampus as well as to other OARs on WBRT.

The objective of the present study is to compare the dose sparing capability of dac-VMAT and sapf-VMAT at hippocampus during WBRT.

\section{Methodology}

Patient selection and computed tomography simulation

Twenty patients, who had been previously treated with WBRT in 2012-2019, were randomly selected and enrolled in the present study. All patients had a previous primary cancer diagnosis that had metastasized and infiltrated the brain. Written consent was obtained from each patient for the present study.

All patients were simulated in the supine position. TIMO Head \& Neck Support Cushions (Med-Tec, Orange City, IA) and thermoplastic mask (Klarity Medical \& Equipment Co. Ltd., Guangzhou, China) were

Table 1 Dose criteria of RTOG 0933 protocol. Dose prescription of $30 \mathrm{~Gy}$ in 10 fractions

\begin{tabular}{llll}
\hline RTOG 0933 protocol & Per protocol & Acceptable variation & Unacceptable deviation \\
\hline Whole brain PTV & $D_{2 \%}<37.5 \mathrm{~Gy}$ & $\mathrm{D}_{2 \%}=37.5 \mathrm{~Gy}$ & $\mathrm{D}_{2 \%}>40 \mathrm{~Gy}$ \\
& $\mathrm{D}_{98 \%}>25 \mathrm{~Gy}$ & $\mathrm{D}_{98 \%}<25 \mathrm{~Gy}$ & $\mathrm{~V}_{30}<90 \%$ \\
Hippocampus & $\mathrm{D}_{100 \%}<9 \mathrm{~Gy}$ & $\mathrm{D}_{100 \%}=10 \mathrm{~Gy}$ & $\mathrm{D}_{100 \%}>10 \mathrm{~Gy}$ \\
Optic nerves and optic chiasm & $\mathrm{D}_{\max }<16 \mathrm{~Gy}$ & $\mathrm{D}_{\max }=17 \mathrm{~Gy}$ & $\mathrm{D}_{\max }>17 \mathrm{~Gy}$ \\
\hline
\end{tabular}


used for immobilization. The computed tomography (CT) simulation images (native, $120 \mathrm{kV}, 80 \mathrm{~mA}$, slice thickness $3 \mathrm{~mm}$, in-plane resolution $1 \mathrm{~mm}$ ) were acquired using dual-source CT scanner (SOMATOM Definition, Siemens Healthcare, Forchheim, Germany). CT simulation images were stored as Digital Imaging and Communications in Medicine images and were electronically transferred to the Eclipse ${ }^{\text {ma }}$ (Varian Medical System, Palo Alto, CA) version 15.5 treatment planning system for WBRT planning.

\section{Target delineation}

The selected patients' treatment plans were retrieved and re-planned for this retrospective planning study. CT simulation images of each patient were co-registered with the most recent T1-weighted cranial magnetic resonance (MR) images (contrast medium-enhanced base, slice thickness $3 \mathrm{~mm}$, in-plane resolution $0.8 \mathrm{~mm}$ ) with reference to the bony anatomy. The eyes, lenses, optic nerves, optic chiasm, brainstem and hippocampus were defined as OARs. The hippocampus was manually delineated by single radiation oncologist strictly following the RTOG 0933 atlas definition (available at: http://www. rtog.org). A hippocampal Planning Risk Volume (PRV) was defined as the hippocampus plus uniform $5 \mathrm{~mm}$ margin using inbuilt margin expansion function [16]. The whole brain PTV for optimization was created by delineating the whole brain and excluding the hippocampal PRV.

\section{Dose prescription}

The treatment prescription to the whole brain PTV was set to deliver $30 \mathrm{~Gy}$ over the course of 10 fractions. All VMAT plans were normalized to ensure that $97 \%$ of the whole brain PTV was covered by $95 \%$ of the prescribed dose. The acceptable compliance criteria for whole brain PTV and OARs planning doses were listed in Table 1 following RTOG 0933 protocol.

\section{Treatment planning}

All VMAT plans (RapidArc ${ }^{\mathrm{Tm}}$, Varian Medical System, Palo Alto, CA) were optimized using Eclipse $^{\mathrm{mt}}$ (Varian Medical System, Palo Alto, CA) version 15.5 treatment planning system. A total of 40 treatment plans (20 dacVMAT plans and 20 sapf-VMAT plans) were produced in the present study. Plans were scheduled using 6-MV photon beams with a maximum dose rate of $600 \mathrm{MU} /$ min on a Varian TrueBeam ${ }^{\mathrm{Tm}}$ linear accelerator with a Millennium 120-leaf MLC (Varian Medical Systems, Palo Alto, CA). Jaw tracking was enabled. The Photon Optimizer (PO, ver.15.5.11, Varian Medical Systems) was used for VMAT optimization. Optimization objectives of major structures were standardized for each technique and were shown in Fig. 1. To avoid introducing bias, the optimization objectives were not modified or individualized between patients of each technique. For dose calculation, the anisotropic analytic algorithm (AAA, ver.15.5.11, Varian Medical Systems) was used with a dose calculation grid of $1 \mathrm{~mm}$. The planning time was similar for each treatment plan in both techniques.

\section{Dual-arc conventional VMAT (dac-VMAT)}

The dac-VMAT plans comprised 2 coplanar arcs of $359.8^{\circ}$ each. Collimator rotation of $30^{\circ}$ and $330^{\circ}$ were used with reference to previous studies [19-21]. The isocentre was placed at the center point equidistant from both hippocampi. The maximum dose rate for the arcs was set to $600 \mathrm{MU} / \mathrm{min}$. Field size was opened up so that the whole brain PTV was completely covered (Fig. 2).

\section{Split-arc partial-field VMAT (sapf-VMAT)}

Four arcs of $179.9^{\circ}$ each were used with the same isocentre as the dac-VMAT plans. The maximum dose rate for the arcs was set to $600 \mathrm{MU} / \mathrm{min}$. Collimator angles were chosen to facilitate better use of the MLC. In the present study, collimator angle of $85^{\circ}, 95^{\circ}, 15^{\circ}$ and $345^{\circ}$ were chosen for field 01, 02, 03 and 04 respectively. Field size of each beam arc was reduced so as to allow the MLC to block the centrally located hippocampus without sacrificing the whole brain PTV coverage (Fig. 3):

(1) Field 01 and field 02: Due to the larger volume of the superior part of whole brain PTV, two field arcs $\left(359.8^{\circ}\right)$ were used to deliver radiation dose. The length of X1 collimator was reduced to $2-3 \mathrm{~cm}$, so that the hippocampus was included. X2 collimator was opened up so that the rest of the superior part of whole brain PTV was covered.

(2) Field 03: The field aimed to deliver radiation dose to the right hemisphere of whole brain PTV. The length of X2 collimator was reduced to $2-3 \mathrm{~cm}$, while the X1 collimator was opened up, so that the entire right hemisphere of whole brain PTV and the right hippocampi were included. Rotational asymmetry of field 03 was compensated by field 04 .

(3) Field 04: The field aimed to deliver radiation dose to the left hemisphere of whole brain PTV. The length of X1 collimator was reduced to $2-3 \mathrm{~cm}$, while the X2 collimator was opened up, so that the entire left hemisphere of whole brain PTV and the left hippocampi were included.

\section{Treatment planning evaluation and quality assurance} With reference to the RTOG 0933 protocol criteria, dosimetric parameters of the both VMAT techniques were extracted and compared. The volume of whole 


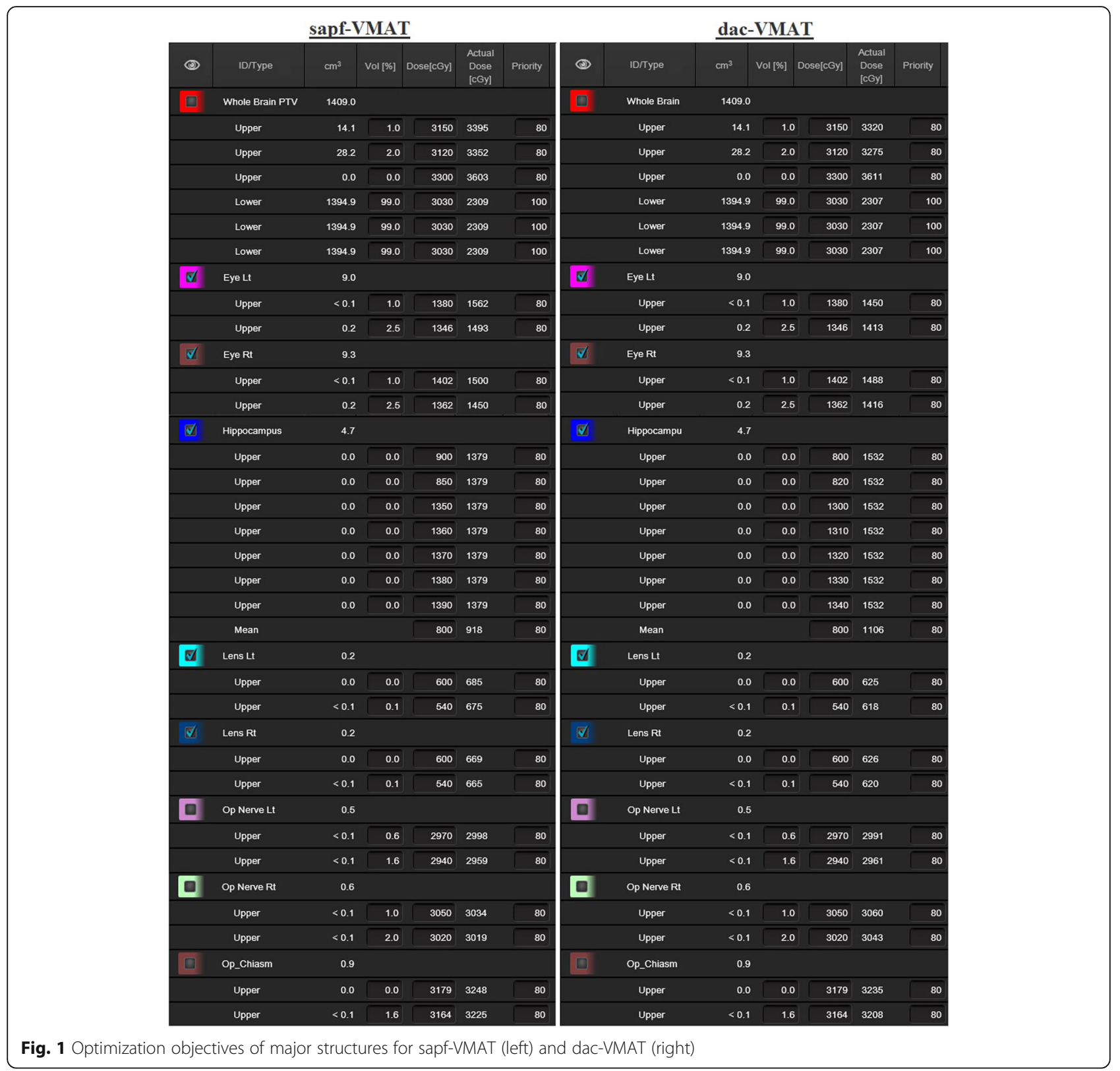

brain PTV receiving 30 Gy $\left(\mathrm{V}_{30 \mathrm{~Gy}}\right)$ was recorded for each plan. Dose homogeneity was quantified in terms of homogeneity index (HI), which was defined in the International Commission on Radiation Units and Measurements Report 83 [30] as follows (Eq. 1).

$$
H I=\frac{\left(D_{2 \%}-D_{98 \%}\right)}{D_{50 \%}}
$$

HI values close to 0 indicated superior homogeneity. Therefore, it was recommended to minimize HI values so as to correspond to more homogeneous dose distribution across the whole brain PTV.
In the present study, the dosimetric parameters of OARs were extracted for comparison including minimum, maximum and mean $\left(D_{\text {mean }}\right)$ doses to the hippocampus; the maximum and mean doses to the eyes, and maximum doses to the optic nerves, optic chiasm, and lenses. Total monitor unit (MU) of each plan was collected and compared between both VMAT techniques. Quality assurance (QA) of treatment plans were performed by dose calculation verification system - MobiusCalc version 2.1 (Mobius Medical Systems, LP, Houston, TX). Treatment plans were exported to MobiusCalc and re-calculated in the patient CT using independently verified beam models and a Graphical Processing Units (GPU)-accelerated collapsed-cone dose 


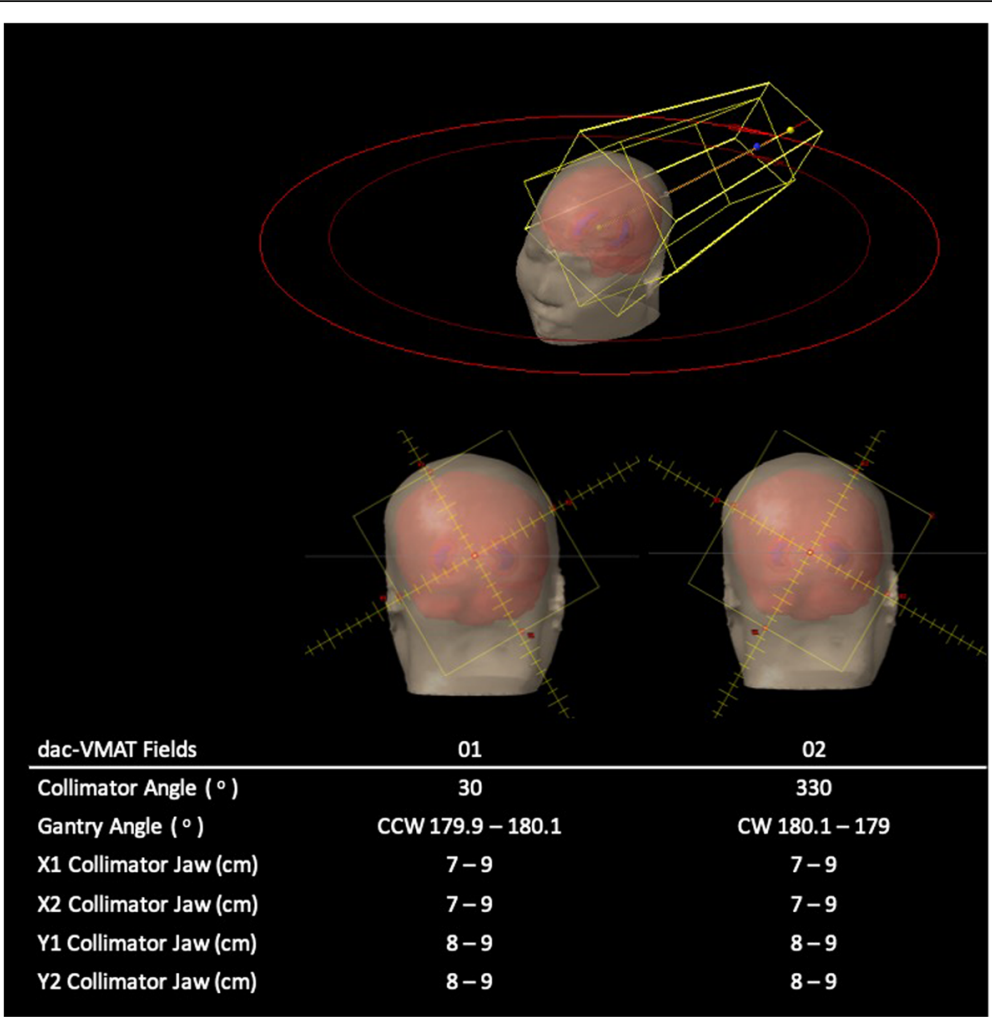

Fig. 2 Beam arrangement of dac-VMAT (CCW = counterclockwise; CW = clockwise)

algorithm. Target dose, DVH limits, 3D gamma, and deliverability of all treatment plans were verified. All treatment plans were required to have a gamma value $>95 \%$ with tolerance for distance to agreement as $3 \mathrm{~mm}$ and dose difference as $3 \%$.
Long treatment delivery time has been associated with increased intrafraction motion [31]. In order to investigate both techniques in this respect, beam-on time and delivery time of a single fraction of WBRT with hippocampal sparing were recorded for dac-VMAT and sapf-

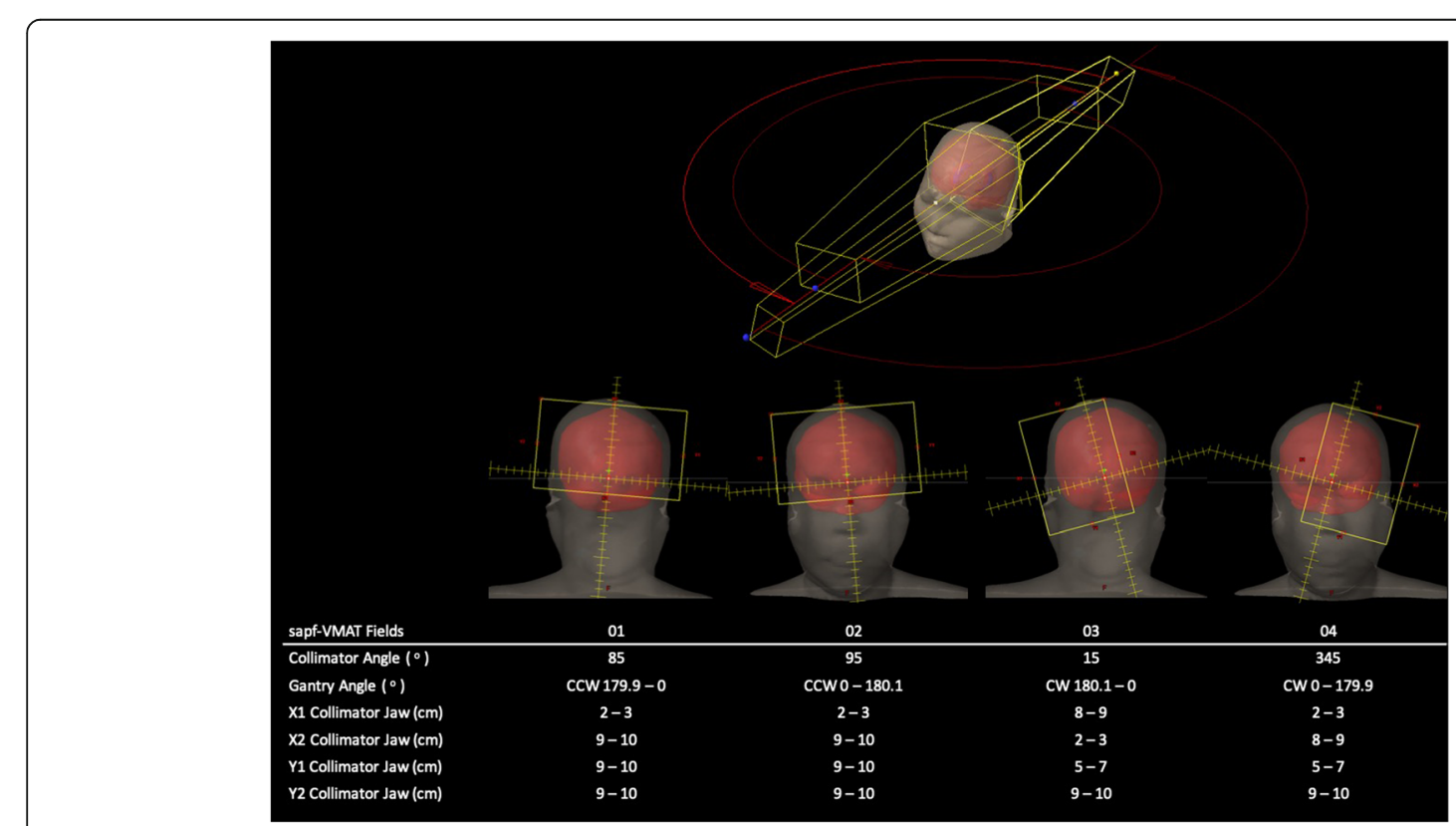

Fig. 3 Beam arrangement of sapf-VMAT (CCW = counterclockwise; $C W=$ clockwise) 
Table 2 Averaged results and comparison of dosimetric parameters using dac-VMAT and sapf-VMAT. Each value was calculated based on the data from 20 patients and was expressed as mean \pm standard deviation (SD)

\begin{tabular}{|c|c|c|c|c|}
\hline Structures & Dosimetric Parameters & dac-VMAT & sapf-VMAT & $p$-value \\
\hline \multirow[t]{4}{*}{ Whole Brain PTV } & $V_{30 G y}(\%)$ & $94.67 \pm 0.25$ & $94.79 \pm 0.13$ & $p=0.358$ \\
\hline & $D_{2 \%}(G y)$ & $33.04 \pm 0.32$ & $33.12 \pm 0.34$ & $p=0.842$ \\
\hline & $\mathrm{D}_{98 \%}(\mathrm{~Gy})$ & $26.11 \pm 0.06$ & $25.84 \pm 0.03$ & $p=0.401$ \\
\hline & $\mathrm{HI}$ & $0.22 \pm 0.02$ & $0.23 \pm 0.01$ & $p=0.435$ \\
\hline \multirow[t]{3}{*}{ Hippocampus } & $D_{100 \%}(G y)$ & $9.23 \pm 0.13 * *$ & $7.86 \pm 0.08 * *$ & $p<0.001$ \\
\hline & $D_{\max }(G y)$ & $16.33 \pm 0.63 * *$ & $13.23 \pm 0.46 * *$ & $p<0.001$ \\
\hline & $D_{\text {mean }}(G y)$ & $10.85 \pm 0.21 * *$ & $9.16 \pm 0.10 * *$ & $p<0.001$ \\
\hline Left Optic Nerve & $\mathrm{D}_{\max }(\mathrm{Gy})$ & $30.80 \pm 0.66$ & $31.23 \pm 0.48$ & $p=0.791$ \\
\hline Right Optic Nerve & $D_{\max }(G y)$ & $30.82 \pm 0.55$ & $30.51 \pm 0.61$ & $p=0.567$ \\
\hline Optic Chiasm & $D_{\max }(G y)$ & $32.36 \pm 0.26$ & $32.48 \pm 0.25$ & $p=0.939$ \\
\hline \multirow[t]{2}{*}{ Left Eye } & $D_{\max }(G y)$ & $16.83 \pm 0.75$ & $17.12 \pm 0.47$ & $p=0.481$ \\
\hline & $D_{\text {mean }}(G y)$ & $10.46 \pm 0.56 *$ & $9.34 \pm 0.32 *$ & $p=0.026$ \\
\hline \multirow[t]{2}{*}{ Right Eye } & $\mathrm{D}_{\max }(\mathrm{Gy})$ & $17.26 \pm 0.63$ & $17.21 \pm 0.26$ & $p=0.991$ \\
\hline & $D_{\text {mean }}(G y)$ & $10.36 \pm 0.46 *$ & $9.07 \pm 0.33 *$ & $p=0.042$ \\
\hline Left Lens & $D_{\max }(G y)$ & $7.32 \pm 0.54$ & $7.33 \pm 0.25$ & $p=0.679$ \\
\hline Right Lens & $D_{\max }(G y)$ & $7.53 \pm 0.44$ & $7.12 \pm 0.41$ & $p=0.985$ \\
\hline Total MU & & $919.69 \pm 130.95$ & $1085.58 \pm 153.57$ & $p=0.053$ \\
\hline Beam-on time (minute) & & $3.14 \pm 0.11$ & $3.04 \pm 0.22$ & $p=0.446$ \\
\hline Delivery time (minute) & & $3.41 \pm 0.11$ & $3.62 \pm 0.22$ & $p=0.437$ \\
\hline
\end{tabular}

${ }^{*} p<0.05$;

$* * p<0.005$ (paired t-test)

VMAT. The beam-on time was defined as the summation of the time elapsed from each treatment field beamon to its beam-off. The delivery time was defined as the time elapsed from the moment of first treatment field beam-on to the end of the last treatment field beam-off, including the time that the gantry travels to the designated starting point. Both beam-on time and delivery time did not include pre-treatment patient setup and daily imaging procedures. Beam-on time and delivery time were measured during QA delivery of the treatment plans.

\section{Statistical analyses}

Statistical comparison between treatment plans of both VMAT techniques were performed using paired t-test. All statistical analyses were performed using SPSS Version 25 statistical software (IBM, USA). $p$-values of $<0.05$ were considered to be statistically significant.

\section{Results}

The QA of all treatment plans showed good correlation and reached a passing rate of 95\% between treatment planning system-calculated dose and QA system calculated dose (Distance to agreement $<3$ $\mathrm{mm}$ and dose difference $<3 \%$ ). Results of dosimetric analysis of whole brain PTV and OARs for the 20 patients in the present study were summarized as mean \pm standard deviation (SD) (Table 2). The mean dose-volume histograms (DVH) of the whole brain PTV (Fig. 4) and OARs (Fig. 5) using dac-VMAT and sapf-VMAT were compared.

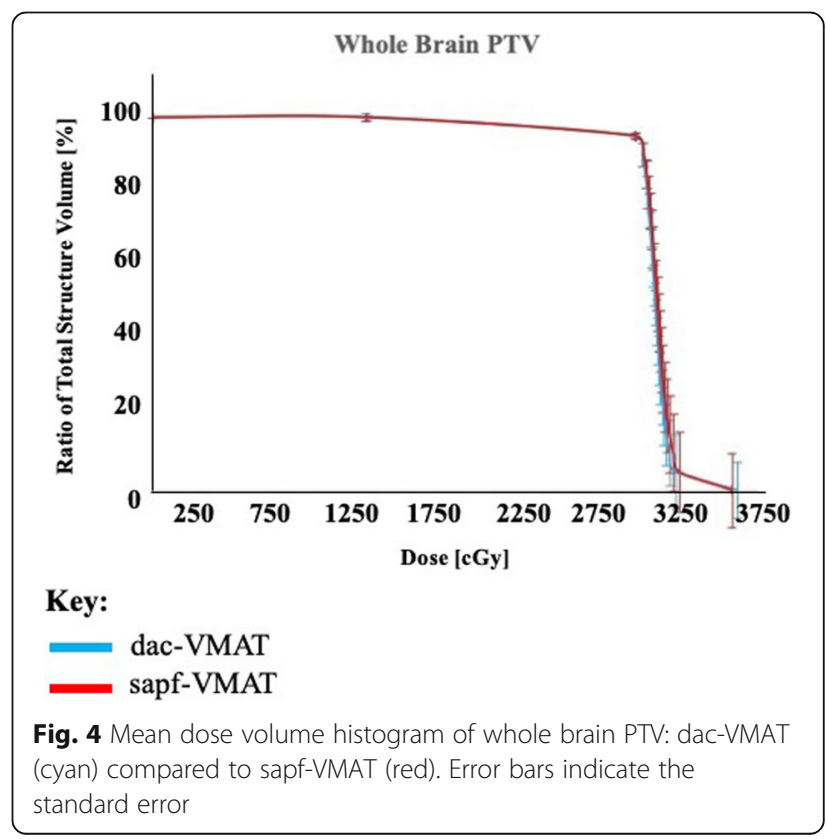




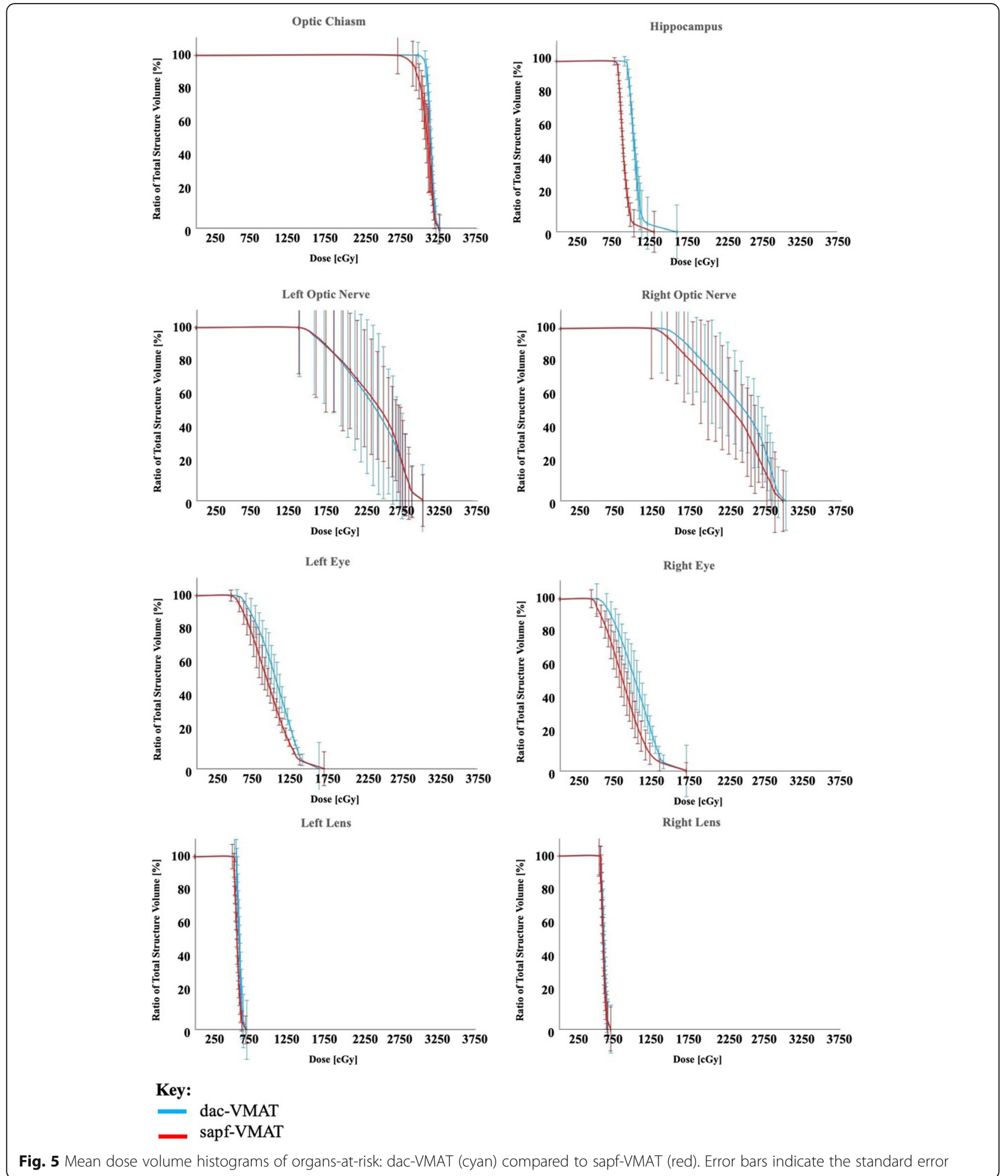

Target coverage and dose homogeneity

The typical dose distribution color washes from 20 Gy to $37.5 \mathrm{~Gy}$ of both treatment techniques were demonstrated in Fig. 6. In the present study, all treatment plans had maximum dose less than $37.5 \mathrm{~Gy}$ as per RTOG 0933 protocol. All treatment plans were capable to produce adequate target coverage. In terms of the whole brain PTV coverage across the 2 treatment techniques, sapfVMAT provided average $\mathrm{V}_{30 \mathrm{~Gy}}$ of $94.79 \%$, which was comparable to dac-VMAT (94.67\%). There were no 


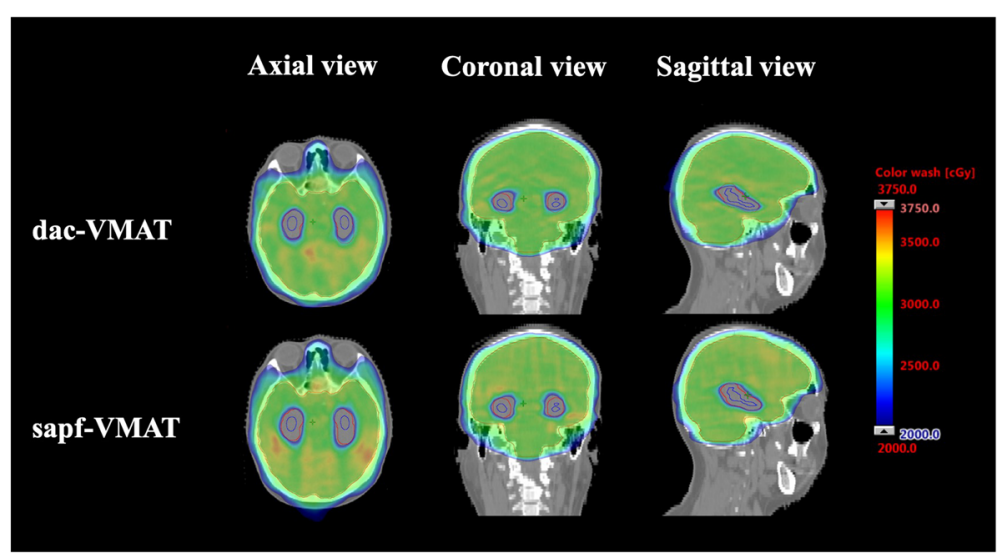

Fig. 6 Dose color wash diagrams of dac-VMAT and sapf-VMAT in axial (left), coronal (middle), and sagittal (right) view

significant differences $(p>0.05)$ between sapf-VMAT vs. dac-VMAT in $\mathrm{V}_{30 \text { Gy. }}$ sapf-VMAT had a mean $\mathrm{HI}$ of 0.23 , compared to 0.22 for dac-VMAT. No significant differences $(p>0.05)$ were found between both techniques. These findings indicated that both treatment techniques in the present study have similar effectiveness in achieving target coverage and dose homogeneity.

\section{Hippocampus}

sapf-VMAT (7.86Gy, $p<0.001$ ) had a significantly lower average $\mathrm{D}_{100 \%}$ compared to dac-VMAT (9.23 Gy). A decrease in hippocampus $D_{\max }$ using sapf-VMAT (13.23 Gy, $p<0.001)$ was statistically significant when compared to dac-VMAT $(16.33 \mathrm{~Gy})$. The resulting mean dose to the hippocampus were $9.16 \mathrm{~Gy}$ for the sapfVMAT. The mean dose of sapf-VMAT was significantly lower than dac-VMAT (10.85 Gy, $p<0.001)$.

\section{Optic chiasm, optic nerves, eyes and lenses}

The average maximum doses to optic chiasm in dacVMAT and sapf-VMAT were $32.36 \mathrm{~Gy}$ and $32.48 \mathrm{~Gy}$ respectively. No significant differences in optic chiasm $\mathrm{D}_{\max }$ were found between both techniques in the present study $(p>0.05)$. In terms of the averaged maximum doses for both optic nerves, sapf-VMAT were comparable to dac-VMAT $(p>0.05)$. In both eyes, sapf-VMAT demonstrated significantly lower $\mathrm{D}_{\text {mean }}$ compared to dac-VMAT $(p<0.05)$. No significant differences in $D_{\max }$ of both eyes and lenses were found between dac-VMAT and sapf-VMAT $(p>0.05)$.

Total monitor unit, beam on time and delivery time The average total MU in dac-VMAT and sapf-VMAT were 919.69 and 1085.58 respectively. The averaged beam-on time were $3.14 \mathrm{~min}$ and $3.04 \mathrm{~min}$ for dacVMAT and sapf-VMAT respectively, while the averaged treatment delivery time were $3.41 \mathrm{~min}$ and $3.62 \mathrm{~min}$ respectively. No significant differences $(p>0.05)$ were found between both techniques for beam-on time and delivery time.

\section{Discussion}

In this planning study, two different techniques (dacVMAT and sapf-VMAT) were compared in the treatment of 20 patients with brain metastases. All treatment plans were able to achieve the acceptable range of RTOG 0933 (Table 1). Radiation dose to the hippocampus and other OARs were reduced while the whole brain PTV coverage was not compromised.

The present study has suggested a radiation therapy technique - sapf-VMAT, which has consistently demonstrated lower hippocampus dose compared to dacVMAT plans, with an average reduction of around $14.84,18.98$ and $15.58 \%$ in $\mathrm{D}_{100 \%}, \mathrm{D}_{\max }$ and $\mathrm{D}_{\text {mean }}$ of hippocampus respectively. Meanwhile, hippocampus $\mathrm{D}_{100 \%}$ and $\mathrm{D}_{\max }$ have been reduced to an average of 7.86 Gy and 13.23 Gy in sapf-VMAT, which are less than the cutoff value of radiation induced neurocognitive deficit onset as described by Gondi et al. [16].

In the present study, dac-VMAT technique comprises 2 coplanar full arcs with large field size covering the whole brain with reference to previous published studies [19-21]. The large irradiation field of whole brain PTV requires a larger jaw opening. This technique may produce a substantial low dose volume in the hippocampus, as a consequence of multi-leaf collimator (MLC) leakage and scatter radiation. The limitation of MLC movement in large field size dac-VMAT may also induce the island blocking problem [32, 33] (Fig. 7). The island blocking problem exists when $\geq 2$ areas of whole brain PTV share the same MLC leaf pair, resulting in an area of hippocampus that is not blocked by the MLC, and hence increased low dose spillage to the hippocampus. Since reduced field size in either X1 and X2 collimator jaw has been employed for sapf-VMAT plans, an independent jaw can be moved to block off part 


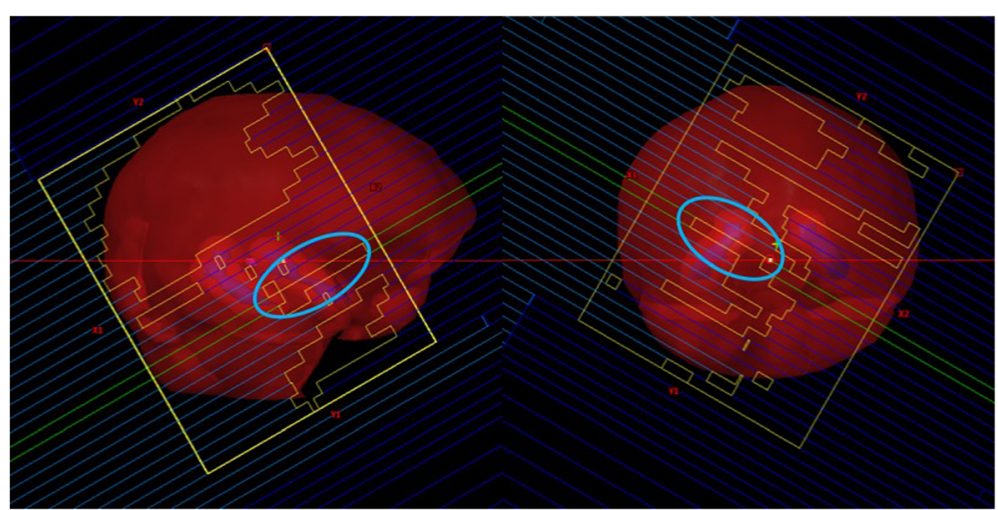

Fig. 7 The island blocking problem exists in dac-VMAT that resulting in an area of hippocampus that is not blocked by the MLC (cyan colour circle)

of the field to reduce scatter radiation. This feature is useful for adjacent normal healthy tissue sparing, that is, the hippocampus. In addition, the reduced field size can shorten travelling distance of MLC, and therefore MLC movement is less likely to be restricted by its velocity and physical limitation. Thus, MLC in sapf-VMAT are capable of shielding the hippocampus in all gantry angles, while remaining enough dose coverage to whole brain PTV.

In the coplanar VMAT planning, constraint of radiation dose to the eyes and hippocampus is sometimes considered to be a difficult goal. Since the eyes and the hippocampus are collated on the same plane, which creates difficulties during optimization using dac-VMAT. In sapf-VMAT plans, the proposed split-arc design can help the optimizer to avoid irradiation of whole brain PTV with the expense of hippocampus and both eyes by collimator rotation between the arcs. The reduced treatment field size can also reduce the swept angle that both eyes lie within the treatment field, resulting in dose reduction in hippocampus and both eyes using sapfVMAT.

In addition to tissue sparing, another major advantage of using sapf-VMAT is that the overall swept angle remains equal to the dac-VMAT for WBRT (i.e. $719.6^{\circ}$ ), although the number of treatment arcs in sapf-VMAT is increased to 4 . Hence, no major increment in treatment delivery time (13 s more than dacVMAT on average) is induced using sapf-VMAT. This technique will not impact on patient comfort on the treatment couch and affect the reproducibility of treatment position.

The sapf-VMAT plans generated in this study has resulted in higher averaged MU usage than the dacVMAT plans (averaged difference of $166 \mathrm{MU}$ ). It is believed that the higher MU usage resulting from sapfVMAT plans is a consequence of the highly conformal dose distributions and superior OAR sparing.
Admittedly, higher MU has its drawbacks such as the potential increase in total body dose because of scattering and leakage from MLC. Therefore, in future improvement of the sapf-VMAT, efforts should be spent on reducing the $\mathrm{MU}$ usage while maintaining the plan quality.

\section{Conclusion}

The present study has proposed a radiation therapy technique, namely sapf-VMAT, that has employed splitarc and reduced field size. This technique has demonstrated significant dose reduction to the hippocampus and eyes compared to dac-VMAT. Therefore, the clinical usability and functional outcome of this strategy should be further investigated in sapf-VMAT.

\section{Acknowledgements}

The authors would like to thank the editor and two anonymous reviewers for their constructive comments. The authors would also like to thank the Oncology Centre, St. Teresa's Hospital (HKSAR) for providing access to the treatment planning system and patient data. Sincere appreciation is also extended to Dr. Gordon Kwok Hung Au, Dr. Thomas Man Cheuk Ng, Mr. Yan Kit Wah, Mr. To Wing Mok, Ms. Tik Yan Nam and Dr. Wincy Wing Sze Wong for their assistance in data collection and comments on dosimetric planning. Special thanks to Mr. Chu King-Shan for English editing of this manuscript.

\section{Authors' contributions}

Conception and design of the study - Adams HL Yuen, Alex KL Li. Acquisition of data - Adams HL Yuen, Alex KL Li, Philip CY Mak. Analysis and interpretation of data - Adams HL Yuen, Philip CY Mak. Drafting and revising the article - Adams HL Yuen, PM Wu.

Final approval of the manuscript - Alex KL Li, PM Wu, Adams HL Yuen.

\section{Funding}

The authors declare no competing financial interests.

\section{Availability of data and materials}

The data that support the findings of this study are available from the Oncology Centre, St. Teresa's Hospital (HKSAR) but restrictions apply to the availability of these data, which were used under permission for the current study, and so are not publicly available. Data are however available from the authors upon reasonable request and with permission of the Oncology Centre, St. Teresa's Hospital (HKSAR) at the following e-mail address: sthochk@gmail.com. 


\section{Ethics approval and consent to participate}

All procedures in this study were reviewed and approved by the University of Hong Kong and the Oncology Centre, St. Teresa's Hospital (HKSAR).

\section{Consent for publication}

Publication of this study was approved by the University of Hong Kong and the Oncology Centre, St. Teresa's Hospital (HKSAR).

\section{Competing interests}

None.

Received: 5 November 2019 Accepted: 10 February 2020 Published online: 18 February 2020

\section{References}

1. Kondziolka D, Patel A, Lunsford LD, Kassam A, Flickinger JC. Stereotactic radiosurgery plus whole brain radiotherapy versus radiotherapy alone for patients with multiple brain metastases. Int J Radiat Oncol Biol Phys. 1999; 45:427-34.

2. Andrews DW, Scott CB, Sperduto PW, Flanders AE, Gaspar LE, Schell MC, et al. Whole brain radiation therapy with or without stereotactic radiosurgery boost for patients with one to three brain metastases: phase III results of the RTOG 9508 randomised trial. Lancet. 2004;363:1665-72.

3. Péchoux $\mathrm{CL}$, Arriagada R. Prophylactic cranial irradiation in small cell lung cancer. Hematol Oncol Clin North Am. 2004;18:335-72.

4. Slotman B, Faivre-Finn C, Kramer G, Rankin E, Snee M, Hatton M, et al. Prophylactic cranial irradiation in extensive small-cell lung cancer. N Engl J Med. 2007:357:664-72.

5. Gondi V, Tolakanahalli R, Mehta MP, Tewatia D, Rowley H, Kuo JS, et al. Hippocampal-sparing whole-brain radiotherapy: a "how-to" technique using helical tomotherapy and linear accelerator-based intensity-modulated radiotherapy. Int J Radiat Oncol Biol Phys. 2010;78:1244-52.

6. DeAngelis LM, Delattre JY, Posner JB. Radiation-induced dementia in patients cured of brain metastases. Neurology. 1989;39:789.

7. Fisher B, Seiferheld W, Schultz C, DeAngelis L, Nelson D, Schold SC, et al, Secondary analysis of radiation therapy oncology group study (RTOG) 9310: an intergroup phase II combined modality treatment of primary central nervous system lymphoma. J Neuro-Oncol. 2005;74:201-5.

8. Gavrilovic IT, Hormigo A, Yahalom J, DeAngelis LM, Abrey LE. Long-term follow-up of high-dose methotrexate-based therapy with and without whole brain irradiation for newly diagnosed primary CNS Iymphoma. J Clin Oncol. 2006;24:4570-4.

9. Welzel G, Fleckenstein K, Schaefer J, Hermann B, Kraus-Tiefenbacher U, Mai SK, et al. Memory function before and after whole brain radiotherapy in patients with and without brain metastases. Int J Radiat Oncol Biol Phys. 2008;72:1311-8

10. Chang EL, Wefel JS, Hess KR, Allen PK, Lang FF, Kornguth DG, et al. Neurocognition in patients with brain metastases treated with radiosurgery or radiosurgery plus whole-brain irradiation: a randomised controlled trial. Lancet Oncol. 2009;10:1037-44.

11. Barani IJ, Benedict SH, Lin PS. Neural stem cells: implications for the conventional radiotherapy of central nervous system malignancies. Int J Radiat Oncol Biol Phys. 2007;68:324-33.

12. Kirby N, Chuang C, Pouliot J, Hwang A, Barani IJ. Physics strategies for sparing neural stem cells during whole-brain radiation treatments. Med Phys. 2011;38(10):5338-44.

13. Belarbi K, Jopson T, Arellano C, Fike JR, Rosi S. CCR2 deficiency prevents neuronal dysfunction and cognitive impairments induced by cranial irradiation. Cancer Res. 2013;73:1201-10.

14. Greene-Schloesser D, Moore E, Robbins ME. Molecular pathways: radiationinduced cognitive impairment. Clin Cancer Res. 2013;19:2294-300.

15. Gutiérrez AN, Westerly DC, Tomé WA, Jaradat HA, Mackie TR, Bentzen SM, et al. Whole brain radiotherapy with hippocampal avoidance and simultaneously integrated brain metastases boost: a planning study. Int J Radiat Oncol Biol Phys. 2002;69:589-97.

16. Gondi V, Pugh SL, Tome WA, Caine C, Corn B, Kanner A, et al. Preservation of memory with conformal avoidance of the hippocampal neural stem-cell compartment during whole-brain radiotherapy for brain metastases (RTOG 0933): a phase II multi-institutional trial. J Clin Oncol. 2014;32:3810.
17. Limoli CL, Giedzinski E, Rola R, Otsuka S, Palmer TD, Fike JR. Radiation response of neural precursor cells: linking cellular sensitivity to cell cycle checkpoints, apoptosis and oxidative stress. Radiat Res. 2004;161:17-27.

18. Gondi V, Hermann BP, Mehta MP, Tomé WA. Hippocampal dosimetry predicts neurocognitive function impairment after fractionated stereotactic radiotherapy for benign or low-grade adult brain tumors. Int J Radiat Oncol Biol Phys. 2012;83:e487-93.

19. Rong Y, Evans J, Xu-Welliver M, Pickett C, Jia G, Chen Q, et al. Dosimetric evaluation of intensity-modulated radiotherapy, volumetric modulated arc therapy, and helical tomotherapy for hippocampal-avoidance whole brain radiotherapy. PLoS One. 2015;10:e0126222.

20. Pokhrel D, Sood S, Lominska C, Kumar P, Badkul R, Jiang H, et al. Potential for reduced radiation-induced toxicity using intensity-modulated arc therapy for whole-brain radiotherapy with hippocampal sparing. J Appl Clin Med Phys. 2015;16:131-41.

21. Sood S, Pokhrel D, McClinton C, Lominska C, Badkul R, Jiang H, et al. Volumetric-modulated arc therapy (VMAT) for whole brain radiotherapy: not only for hippocampal sparing, but also for reduction of dose to organs at risk. Med Dosim. 2017;42:375-83.

22. Rossi M, Boman E, Skyttä T, Kapanen M. A novel arc geometry setting for pelvic radiotherapy with extensive nodal involvement. J Appl Clin Med Phys. 2016:17:73-85.

23. Shen J, Bender E, Yaparpalvi R, Kuo HC, Basavatia A, Hong L, et al. An efficient volumetric arc therapy treatment planning approach for hippocampal-avoidance whole-brain radiation therapy (HA-WBRT). Med Dosim. 2015:40:205-9.

24. Wang K, Tobillo R, Mavroidis P, Pappafotis R, Pearlstein KA, Moon DH, et al. Prospective assessment of patient-reported dry eye syndrome after whole brain radiation. Int J Radiat Oncol Biol Phys. 2019;105:765-72.

25. Hsu CR, Tai MC, Chang YH, Chien KH. Rapid onset of radiation maculopathy after whole-brain radiation therapy: a case report. Medicine. 2016;95:e4830.

26. Lai $Y$, Chen $Y$, Wu S, Shi L, Fu L, Ha H, et al. Modified volumetric modulated arc therapy in left sided breast cancer after radical mastectomy with flattening filter free versus flattened beams. Medicine. 2016;95.

27. Huang $B$, Fang Z, Huang Y, Lin P, Chen Z. A dosimetric analysis of volumetric-modulated arc radiotherapy with jaw width restriction vs 7 field intensity-modulated radiotherapy for definitive treatment of cervical cancer. Br J Radiol. 2014;87:20140183.

28. Jang H, Park JY, Kim MH, Chun M, Noh OK, Park HJ, et al. EP-1933: half field VMAT for MLC leakage reduction and dosimetric impact in whole pelvis radiotherapy. Radiother Oncol. 2018;127:S1050-1.

29. Vieillot S, Azria D, Lemanski C, Moscardo CL, Gourgou S, Dubois JB, et al. Plan comparison of volumetric-modulated arc therapy (RapidArc) and conventional intensity-modulated radiation therapy (IMRT) in anal canal cancer. Radiat Oncol. 2010;5:92

30. International Commission on Radiation Units and Measurements Report 83. Prescribing, recording, and reporting photon-beam intensity-modulated radiation therapy (IMRT). J ICRU. 2010:10.

31. Teoh M, Clark CH, Wood K, Whitaker S, Nisbet A. Volumetric modulated arc therapy: a review of current literature and clinical use in practice. $\mathrm{Br} J$ Radiol. 2011;84:967-96.

32. Kang J, Ford EC, Smith K, Wong J, McNutt TR. A method for optimizing LINAC treatment geometry for volumetric modulated arc therapy of multiple brain metastases. Med Phys. 2010;37:4146-54.

33. Wu Q, Snyder KC, Liu C, Huang Y, Zhao B, Chetty IJ. Optimization of treatment geometry to reduce normal brain dose in radiosurgery of multiple brain metastases with single-Isocenter volumetric modulated arc therapy. Sci Rep. 2016;6:34511.

\section{Publisher's Note}

Springer Nature remains neutral with regard to jurisdictional claims in published maps and institutional affiliations. 